\title{
LA ESTRUCTURA AXIOLÓGICA DE LA PERSONALIDAD
}

\section{Psicologia experimental y cientifica}

En la psicología existe una desafortunada combinación entre la inaccesibilidad y dificultad de su materia y su método experimental y experimentador. La personalidad es invisible, no es algo accesible a los sentidos. $Y$, sin embargo, la psicología se ufana de su base científica e identifica el método científico con la observación. Permítaseme citar un tratado muy conocido, Seven Psychologies, de Edna Heidbreder: " "Cuando los estudiantes se ponen a observar más que a reflexionar sobre el material psicológico -aunque difieran en su definición formal de él-, cuando ven su materia como parte del mundo natural, cuando intentan señalar con ayuda de métodos experimentales y cuantitativos las condiciones en las que ocurren o no los procesos psicológicos, allí empieza la psicología como ciencia". Pero cuando este procédimiento se sigue hasta su fin lógico, como en el conductismo, la personalidad desaparece. Leemos en un diccionario alemán de psicología, ${ }^{2}$ bajo el rubro de "Personalidad" (Persönlichkeit): "La cualidad peculiar de un individuo, la totalidad de todas sus manifestaciones, inmediatas o mediatas, de procesos psíquicos y propiedades humanas"; "la personalidad es lo que señala el carácter distintivo, individual y único de una persona". La personalidad es la suma total de todas las posibilidades realizadas con plena conciencia por la persona, el desarrollo consciente de todas sus posibilidades a lo largo de su vida. De modo un tanto filosófico, podríamos decir que la personalidad es la esencia de la persona, lo que hace de la persona la persona que es, y lo que persiste como algo distintivo durante toda su vida. En el conductismo, no hay tal esencia de una persona. La personalidad en cualquier periodo dado es el sistema de hábitos dominante en ese tiempo. ${ }^{3}$ En la psicología conductista, la personalidad no subsiste y persiste a través de las vicisitudes de la vida a la manera de lo teológico." "Así, pues, todo el sistema de conducta, la personalidad completa, está construida a partir de unas cuantas reacciones simples por el proceso de condicionamiento."5 A partir de ellas, el conductista muestra al final "un ser humano completo; de acuerdo con las palabras de Watson, 'una máquina orgánica ensamblada y lista para

1 New York, 1993, Appleton-Century-Crofts, p. 112.

2 Friedrich Dorsch, Psychologisches Wörterbuch, Hamburg-Bern, 1959, Richard Meiner-Hans Huber.

3 Heidbreder, op. cit., pp. 253 ss.

4 lbid.

5 Heidbreder, op. cit., p. 254 . 
moverse" ". 6 "La personalidad no es más que un sistema de reacciones y por ello puede estudiarse con los métodos fácticos de la ciencia. El que no pueda ser estudiada muy completamente en el presente es el resultado del hecho de que aún no se han desarrollado métodos adecuados; nada hay en la personalidad que por necesidad eluda al tratamiento cientifico." 7 En otras palabras, si conocemos bastantes reacciones de un humano, podemos, cuando menos en teoría, componer su personalidad a partir de ellas. Asi, la personalidad se ensambla a partir de sus manifestaciones mínimas mensurables. Pero ¿qué hay detrás de estas manifestaciones?

Vayamos ahora al extremo opuesto del espectro psicológico y veamos cómo considera a la personalidad la psicología existencialista: "La persona. lidad no es naturaleza, no pertenece a la jerarquía objetiva de la naturaleza, como parte subordinada de ella ... EI hombre es una personalidad no por naturaleza, sino por espíritu ... La personalidad es una categoría axiológica, una categoría de valor ... La personalidad debe construirse a sí misma, enriquecerse a sí misma, llenarse a sí misma con un contenido universal, lograr la unidad en la totalidad en toda la extensión de su vida ... El secreto de la existencia de la personalidad yace en su absoluta irremplazabilidad, su acontecer sólo una vez, su unicidad, su incompatibilidad. Todo lo individual es irremplazable ... La personalidad no es una categoría biológica o psicológica sino ética y espiritual ... La personalidad no es una parte de la sociedad, del mismo modo que no es una parte de una raza ... Todas las doctrinas sociológicas acerca del hombre son erróneas ... Sólo una filosofía existencial, y no una filosofía sociológica, como tampoco una filosofía biológica o psicológica puede construir la verdadera doctrina del hombre como personalidad."s

Tenemos aquí, pues, los dos extremos de la psicología, el conductista $\mathrm{y}$ el existencialista. $\mathrm{Y}$ el problema es cómo pueden combinarse estos extremos en una sola ciencia. La respuesta es, justo: a causa de que la psicología no es todavía una ciencia. Es una ciencia que está haciéndose, pero no una ciencia hecha. Una ciencia hecha, en el sentido de ciencia exacta, consiste en un marco formal de referencia, tal como las matemáticas, que se aplica a todos los fenómenos del campo en cuestión. Así sucede en la física, la química, la astronomía y otras ciencias exactas. Una ciencia que está haciéndose es una colección de experimentos y se le llama ciencia experimental o empírica. ${ }^{9}$

6 Ibid.

7 Heidbreder, op. cit., p. $25 \%$.

8 Nicolas Berdyaev, Slavery and Freedom, New York, 1944, Scribners, pp. 20-34.

9 En la historia de la psicología se hizo una distinción significativa entre ambas. En la discusión entre Brentano y Wundt, el primero sostuvo que "era necesario distinguir entre el tono que uno oye y el oír un tono. Sostenía que el oir un tono es un proceso o acto psicológico; el tono oido es el contenido del acto". Este último es el rasgo distintivo 
Ambas, las observaciones empíricas y las experimentales, son signos de una ciencia que aún no ha madurado, sino que está todavía en la etapa de recoger material a través de la observación fenomenológica y cuantitativa -es un muestreo del campo, pero no una visión unitaria de él. El muestreo puede convertirse en una ciencia sólo si hay cuando menos un recogedor de muestras que trascienda ese método, metiéndose en su materia a tal grado que vea su totalidad con una sola mirada: el rasgo característico que es su esencia. Así, Goethe vio la Urpflanze mientras paseaba bajo las palmeras de Palermo, Darwin vio el patrón de la evolución mientras leía a Malthus y Newton la ley de la gravitación al sentir el golpe de la famosa manzana. Existe mucha literatura acerca de este tipo de experiencia -llamada algunas veces la experiencia "Ajá"- y el éxtasis o el shock de reconocimiento. Algunas veces este shock es físico, como en el caso de Franklin cuando recibió la descarga eléctrica a través de su cometa, otras veces es una visión tan tremenda que el visionario es incapaz de cumplir su promesa, o sólo es capaz de cumplirla en parte, como Kepler. Por lo común, el primer visionario recibe sólo el shock inicial; a éste le sigue toda una serie de shocks, cada uno de los cuales amplía y profundiza la vista, y seguido cada uno de ellos por un ejército de investigadores secundarios que aprenden a tratar con seguridad y en forma rutinaria lo que consumió la vida del pionero. Así, Franklin llevó a Faraday, Faraday a Maxwell, Maxwell a Marconi y Edison, y éstos últimos a la tienda de aparatos de alta fidelidad de la esquina. Kepler llevó a Newton, Newton a Laplace, Laplace a Einstein y Einstein a Fermi y al almirante Rickover, a la bomba atómica y sus técnicos.

Cada ciencia exacta ha tenido su pionero unificador, ya sea Newton en la astronomía, Galileo en la física, Lavoisier y Mendeleyev en la química, Darwin y Mendel en la biología y la genética, etc. Cada uno de estos hombres creó un sistema omnicomprensivo, con frecuencia matemático, que se aplicaba al campo en cuestión, logró la unificación del campo y la medición e interrelacionalidad de sus datos $-\mathrm{y}$ estos datos fueron muy diferentes

de un proceso psicológico: se refiere a algo que no es él mismo - "tiende" a un objeto. Por lo tanto, un verdadero proceso psicológico es, en primer lugar, acto, no contenido, aun cuando este último sea indispensable para el acto psicológico. Contra esto, Wundt sostenia que es "el contenido sensible del Do menor lo que constituye el proceso psicolbgico; lo único que puede observarse es este contenido, los actos no pueden ser objeto de observación directa y por ello no pueden constituir el material de una ciencia; pero los procesos, como el oír tonos, son contenidos sensibles y lugares comunes en el laboratorio psicológico. El estudio de tales procesos por medio de los métodos aprobados por la ciencia natural debe constituir el trabajo básico de la psicología". Esta diferencia en cuanto a la materia implicaba una diferencia en el método. Los hechos no se prestan para la psicologia experimental. El estudioso de los hechos mentales utiliza la penetración fenomenológica y ésta se ha caracterizado como empírica y opuesta a lo experimental. "Pero la psicología de Brentano no era en manera alguna un retorno a la vieja psicologia metafísica. El método empírico era un método de observación, aunque no de experimentación." (Heidbreder, op. cit., p. 99.) 
de los que recoge una ciencia experimental o empírica. Esta última no tiene un marco de referencia omnicomprensivo y, por ello, sus datos son muestras tomadas al azar. En una ciencia matemática exacta, como han explicado Kant y otros, la recolección de datos está determinada por el marco de referencia de las matemáticas mismas. Como dijo Kant, una ciencia exacta es sintética a priori, en tanto que una ciencia experimental o empírica busca aún su a priori, es decir, su patrón matemático. Así, pues, una ciencia experimental es una colección de métodos al azar, algunos de los cuales pueden ser matemáticos, como, por ejemplo, el análisis fáctico o la extrapolación estadística de hechos observados, pero tales métodos no están unificados por un Método de la ciencia total. Por así decirlo, una ciencia experimental es un conjunto de columnas sin conexión entre sí, en tanto que una ciencia exacta es un templo con columnas, cuyo techo no sólo cubre sino que da forma $y$ estilo a las columnas individuales en un todo completo.

En este sentido, la psicología no es aún una ciencia. Es, como dice Heidbreder, "una ciencia todavía por hacerse. La curiosidad científica, que ha penetrado en tantas formas de la naturaleza, aparece aquí en el acto mismo de tantear su camino a través de una región que apenas ha empezado a explorar, golpeándose contra las barreras, palpando a través de las confusiones, y trabajando ... en un problema que está aún lejos de resolverse. Pues la psicologia es una ciencia que no ha hecho aún su gran descubrimiento. No ha encontrado nada que haga por ella lo que la teoría atómica hizo por la química, lo que el principio de la evolución orgánica por la biología, lo que la ley del movimiento por la física. No se ha descubierto o reconocido aún nada que le proporcione un principio unificador ... La psicología no ha ganado todavía su gran victoria unificadora". ${ }^{10}$ De esta condición de la psicología —su estar incompleta, su juventud, su aspiración hacia una meta aún distante- derivan las teorías psicológicas su significación, los llamados "sistemas", y por ello pueden coexistir dentro de la misma disciplina general. Es justo la incoherencia de la disciplina lo que hace que la psicología incluya tanto al conductismo como el existencialismo, además de las muchas escuelas intermedias, que son más de las siete que menciona Heidbreder. Puede decirse que hay tantas psicologías cuantos psicólogos notables existen.

Así, pues, resulta obvio que la psicología no será una ciencia en el sentido estricto de la palabra hasta que se descubra, y a menos que se haga, un marco de referencia matemático, lógico o, en general, formal, que dé cuenta de ella no por medio de palabras sino de variables, no en prosa sino por medio de símbolos. Es mi creencia, como lo fue hace muchos años la de Susanne Langer, ${ }^{11}$ que la psicologia, como también lo sostuvo Berdyaev, es una ciencia axiológica y, de acuerdo con las palabras de Susanne Langer, "si

10 Heidbreder, op. cit., pp. 425 s. Subrayado nuestro.

11 The Practice of Philosophy, New York, 1930, Henry Holt. 
alguna vez ha de existir una ciencia axiológica, alguien deberá formular los conceptos básicos que harán surgir de golpe la ética, la estética y, posiblemente, la economía. Si el valor constituye realmente un campo definible, entonces todas estas ciencias están relacionadas como la mecánica, la físicoquímica y la electrodinámica están relacionadas por un elaborado sistema de física pura". ${ }^{12}$ La física misma, podría haber continuado Langer, es sólo una de las muchas ciencias matemáticas y, así, la ética, la estética y posiblemente la economía, la psicología, la sociología y otras disciplinas, que hoy en día llamamos humanidades y disciplinas sociales, se unificarian no por un sistema matemático tradicional, sino por un sistema axiológico formal.

Así, el mundo quedaría dividido en dos grandes partes, el mundo de los hechos científicos y el mundo del valor científico. La realidad sensible de los hechos sería entonces sólo una parte del mundo analizado total, y la otra sería su patrón axiológico ordenador. Como en ese caso tendríamos dos patrones --el del hecho y el del valor-, se seguiría que una y la misma realidad, el mundo, aparecería bajo dos aspectos, según el patrón que se aplicara, tal y como una y la misma curva aparece, de acuerdo con el punto de vista, como convexa o cóncava. El mundo de los hechos constituiría, pues, el mundo de los sentidos, tal como es analizado por el patrón formal de las matemáticas; y el mundo del valor, con sus muchas disciplinas, constituiría el mundo no sensible, tal como es analizado por el patrón formal de valoración, llamado axiología formal. He propuesto este último patrón aplicable a las humanidades y las disciplinas sociales, ${ }^{13}$ incluso la psicología. A esta última la defino como la aplicación de valor extrínseco a personas individuales. Esto muestra a cada persona como una clase de funciones y proporciona la ciencia de la psicología - no como una ciencia naturalista, sino como una ciencia axiológica que muestra cómo cumple o no la persona las diversas funciones que presenta. Dentro del campo general definido como psicología _la dimensión axiológica extrínseca aplicada a personas individuales-, todas las tres dimensiones axiológicas (la sistémica, la extrínseca y la intrínseca) pueden usarse de nuevo. Las aplicaciones de estas dimensiones a las diversas funciones proporcionan las diversas psicologías: la dimensión intrínseca lleva a las psicologías de la Gestalt, humanistas y existencialistas; la extrínseca al conductismo; la sistémica a los sistemas formales de psicología como Hull. Al quedar insertadas en un sistema formal, todas estas ciencias perderían su presente carácter arbitrario. ${ }^{14}$

Así, pues, cuando hablo de la estructura axiológica de la personalidad no me refiero tan sólo a un ejercicio de psicología axiológica como una especie de psicología rellena de valor. Me refiero a algo mucho más preciso y

12 Langer, op. cit., p. 209.

13 Robert S. Hartman, La estructura del valor, México, FCE, 1959.

14 Ibid., p. 313 . 
formal: la concepción de la psicología como una ciencia matemática exacta más que como una ciencia experimental.

Desde luego, las matemáticas en cuestión no pueden ser las matemáticas tradicionales de la ciencia natural, sino que deben ser las matemáticas de la ciencia axiológica; no deben ser unas matemáticas aplicadas a la extensión, sino unas matemáticas aplicadas a la intensión o intensidad. Este tipo de matemáticas tiene una historia aún más larga que las de las matemáticas aplicadas a la naturaleza, desde Aristóteles, a través de la Edad Media, hasta Kant, y se continuó, aunque de modo inconsciente, en los primeros experimentadores de la psicología, como Weber, Fechner, Herbart, y en la tradición experimental de la psicología como un todo. La axiología formal usa estas matemáticas intensivas, aplicadas a propiedades, no a cosas, y formaliza así las disciplinas axiológicas, las humanidades y las ciencias sociales actuales - justo como las matemáticas, en el sentido extensional, aplicadas a las cosas, no a las propiedades, formalizaron las ciencias naturales. A fin de entender la estructura axiológica de la personalidad en este sentido, proporcionaré ahora un breve examen de la axiología formal, el método general de valoración y, después, aplicaré esto a la personalidad, a fin de obtener la estructura axiológica de ésta. Permítaseme, sin embargo, decir ya en este punto que los valores no son como los hechos accesibles a la observación sensible, aunque sean reconocibles y cognoscibles con precisión. Por ejemplo, el valor de una pieza musical no está en una ejecución, sino en el patrón total de todas las ejecuciones que es la partitura de la pieza. Del mismo modo, podemos decir que la axiología formal es la partitura de cualquier experiencia axiológica. Es aplicable a la personalidad en particular porque, como hemos visto en la concepción existencialista, tampoco la personalidad es algo sensorialmente accesible.

\section{El patrón de la axiologia formal}

La formalización de la teoría axiológica en un sistema matemático de lógica intensional se realiza por el mismo procedimiento por el que se ha realizado cualquier ciencia matemática: la identificación axiomática del núcleo de un terreno fenoménico con una fórmula que da origen al sistema en cues. tión. Así, en el caso de Galileo, después de penosas observaciones, llegó a su fórmula: "la velocidad es igual a la división del espacio por el tiempo", $v=\frac{e}{t}$; que hoy es obvia para nosotros. Si camino cien kilómetros en dos horas, llevo una velocidad de cincuenta kilómetros por hora. En el caso de Galileo, la línea de división matemática fue una invención que conmovió al mundo. En el campo de la electricidad, tras los experimentos fundamentales de Faraday, siguieron las fórmulas de Maxwell, que dieron origen a la hipo- 
tética naturaleza ondulatoria de la propagación eléctrica, verificada experimentalmente por Hertz y explotada comercialmente por Marconi. En el campo de la astronomía, tras las penosas observaciones y cálculos de Kepler, que llevaron a sus tres leyes, y los fundamentos mecánicos de Galileo, lo mismo que ciertas contribuciones de Huygens, Newton combinó todo esto en una simple fórmula, la de la gravitación, la ley del cuadrado inverso: los cuerpos se atraen recíprocamente con una fuèrza directamente proporcional al producto de sus masas e inversamente proporcional al cuadrado de las distancias (calcula una constante universal $G$ que siguió siendo desconocida por más de medio siglo después de Newton y fue determinada matemáticamente primero por Cavendish en $\left.179^{8}\right)$.

Estas fórmulas no sólo ordenaron amplios campos de la experiencia de observaciones y experimentos, sino que tuvieron otra función igualmente importante. Una vez establecidas, hicieron innecesarias las observaciones ulteriores, ya que el sistema matemático desarrollado a partir de ellas -el único sistema que da cuenta de todo el campo en cuestión- determinó a priori los fenómenos del campo. Esto es algo muy diferente de las matemáticas usadas en las ciencias experimentales que aún no se han unificado, como en la psicología. En este sentido, dice Gordon W. Allport: "Creo que un instrumento de medición en el campo de la personalidad será mucho mejor si se basa en un buen análisis a priori, que si se basa en dimensiones fácticas u otras logradas de modo adventicio". ${ }^{15}$ En la psicología fundada axiológicamente, lo mismo que en la astronomía fundada matemáticamente, los instrumentos de medición como los tests, se basarán no sólo en buenos análisis a priori, sino en un sistema axiométrico a priori aplicable a la realidad psicológica. "¿Cuál es, pues, mi idea personal? - pregunta Allport_-. Creo que tiene que ver con la búsqueda de un sistema teórico, que tenga lugar para la verdad dondequiera que se encuentre, que abarque la totalidad de la experiencia humana y haga plena justicia a la naturaleza del hombre." 16 Podemos condensar estas palabras algo vagas diciendo que el sistema teórico en cuestión tendrá lugar para la verdad de la aplicación por deducción y abarcará la totalidad de los valores humanos -entre ellos la personalidad.

Así, pues, el arte de construir un sistema científico que es a priori una pura construcción del pensamiento, aunque aplicable a la realidad, es la capacidad de enunciar el fenómeno nuclear de un amplio campo de fenómenos en términos de una fórmula matemática. Galileo lo hizo así con respecto a la Velocidad, Newton con respecto a la Fuerza. Y, para dar un ejemplo final, la ciencia de la óptica quedó establecida al identificarse un rayo de luz con una línea recta; siendo esta última un elemento de la geometría y, por ello, toda la geometría, un sistema formal, resultó aplicable

15 The Person in Psychology, Boston, 1969, Beacon Press, p. 393.

16 Ibid., p. 406 . 
a los rayos de luz. Según las palabras del lógico y matemático del siglo xvir, Johann Heinrich Lambert: "Casi toda la óptica se basa en el hecho de que la luz se propaga en una línea recta. Esto basta para que se le aplique toda la geometría. Y nada resulta más natural que la premisa inversa: dondequiera que hay líneas rectas es aplicable la geometría elemental." ${ }^{17} \mathrm{Si}$ generalizamos este principio llegaremos a la conclusión de que el axioma de una ciencia es la identificación de algo no lógico con algo lógico, donde lo no lógico es el núcleo del campo fenoménico y lo lógico es su formulación matemática. Si aplicamos esto al campo del valor, la axiología formal define el valor como un conjunto de propiedades. Cualquier conjunto de propiedades es un valor y un valor es cualquier conjunto de propiedades. Dado que la palabra "conjunto" es un término fundamental de las matemáticas, la teoría matemática de los conjuntos puede aplicarse al valor y obtenemos un sistema matemático de axiología formal. Por ejemplo, valoremos una silla. Digamos que una silla es una estructura cuya altura alcanza mi rodilla, con un asiento y un respaldo; tiene cuatro propiedades: "altura que alcanza la rodilla", "estructura", "asiento", y "respaldo".

¿Cuántos valores tiene la silla? Recordemos que cualquier conjunto de propiedades es un valor. Así, pues, la pregunta es: ¿cuántos conjuntos de propiedades tiene la silla? Es obvio que tiene un conjunto de cuatro propiedades y cuatro conjuntos de una propiedad cada uno. Esto nos da cinco conjuntos, a saber, el conjunto de cuatro y los cuatro conjuntos de una. ¿Cuántos conjuntos de dos propiedades tiene la silla? Seis: los dos primeros, los dos segundos, los dos terceros, el primero y el último, el primero y el tercero, y el segundo y el último. Tenemos ahora, además de los cinco conjuntos previos, seis conjuntos de propiedades más, o sea once conjuntos en total. Por último, debemos preguntarnos cuántos conjuntos de tres propiedades hay en un conjunto de cuatro propiedades. La respuesta es cuatro de tales conjuntos, a saber, los tres primeros, los tres segundos, el primero y las dos últimas propiedades, las dos primeras y la última propiedad. Añadimos ahora cuatro conjuntos a nuestros once y obtenemos quince conjuntos. Esto significa que la silla con sus cuatro propiedades tiene quince diferentes valores. El conjunto de todas las cuatro propiedades significa que la silla tiene todas las propiedades que le es posible tener y, por ello, es una buena silla, en tanto que un conjunto con una sola propiedad significa que la silla carece de tres propiedades de cuatro, y es una silla muy mala. $Y$ un valor de dos propiedades significa que la silla tiene la mitad de sus propiedades y carece de la mitad de ellas y, por ello, es una silla asíasí. En general, una cosa es buena si tiene todas sus propiedades, no es buena si carece de todas sus propiedades menos una, es mediana si tiene más propiedades de

17 Johann Heinrich Lambert, Philosophische Schriften, Hildesheim, 1969, Georg Olms, vol. VII, p. 10. 
las que carece, y es mala si carece de más propiedades de las que tiene y es así-así si tiene tantas propiedades como aquellas de que carece. Sobre esta simple base, tenemos la identificación de algo no lógico, a saber, el valor, con algo lógico, a saber, el conjunto de propiedades, y sobre esta identificación se basa toda la axiología formal. Es un sistema matemático de conjuntos de propiedades, cada uno de los cuales representa un valor.

El cálculo que se aplica aquí es un cálculo combinatorio. Afirma que, en general, si una cosa tiene $n$ propiedades, tiene $2^{n}-1$ conjuntos de propiedades o valores. Si la cosa tiene, como nuestra silla, cuatro propiedades, tiene $2^{4}-1$ conjuntos de propiedades o valores, lo que es quince exactamente. Pero permítasenos tomar dos ejemplos aplicables a la psicología, uno a la psicología industrial y el otro a la fisiológica. Supongamos que la definición de un empleo consiste de diez propiedades o requisitos de empleo. ¿De cuántas maneras puede un empleado cumplir o no con el empleo? De $2^{10}-101023$ maneras. Sólo hay una manera de hacerlo bien, pero hay 385 maneras medianas, 252 maneras de hacerlo asi-asi y 385 maneras de hacerlo mal. En consecuencia, no basta con decir que un trabajador hace su trabajo en forma mediana; debe determinarse cuál de los conjuntos "medianos" de propiedades es el que llena su trabajo - $9,8,7$ o 6 - y qué conjunto dentro de estos conjuntos. Por ejemplo, hay 120 diferentes posibilidades de llenar siete de los diez requisitos de empleo. ¿Cuál de los 120 es el que llena? Este ejemplo nos muestra lo simplista que resulta nuestra psicología industrial si se la mide con la psicología axiológica. Un empleo, o en general cualquier cosa con diez propiedades, tiene ya mil veintitrés valores. Si hubiera más propiedades, el número de los valores aumentaría enormemente. Los catadores franceses de vinos tienen un registro de $15^{8}$ propiedades para catar un vaso de vino de Borgoña. Esto hace que el vaso de Borgoña tenga $2^{158}$ posibles valores, o $3.6 \times 10^{46}$ valores posibles, una cifra astronómica. $Y$ esto es sólo el total de valores posible cuando un experto cata un vaso de Borgoña. Imaginemos a un experto que maneja un Volkswagen, que conoce al revés y al derecho, con sus 20000 partes, digamos 20 ooo propiedades, y que es perfectamente capaz de valorar el automóvil. El número total de valores posibles es $2^{20000}$, una cifra supra-astronómica - y esto se refiere sólo a un Volkswagen, no a un Cadillac. Estos tremendos números muestran el alcance y complejidad de la valoración. Sin embargo, $2^{20000}$ es todavía un número finito. Desde el punto de vista de lo infinito no hay diferencia entre $2,2^{4}, 2^{10000}, 2^{1000} 000 \mathrm{o}$ cualquier otro número finito. La infinitud matemática, $\chi_{0}$, sólo empieza "después" del "último" número contable.

En la psicología axiológica, en especial si lo que queremos es examinar la estructura axiológica de la personalidad, tenemos que tratar con infinidades de números de propiedades. La introducción de números transfini- 
tos resuelve una paradoja que se sigue de dos de nuestras intuiciones fundamentales subyacentes a las decisiones y valoraciones, como explica Robert $\mathbf{S}$. Brumbaugh. ${ }^{18}$ La paradoja surge de nuestra convicción instintiva "de que en tanto que algunos valores se relacionan por adición, otros no. No hay precio en efectivo, ni grado de comodidad que pueda persuadir a Sócrates para que abandone su búsqueda de la sabiduría, o a Beowulf la suya de gloria y honor. Al mismo tiempo, tenemos que comparar, por adición, toda clase de valores, al tomar decisiones; y tenemos una segunda convicción intuitiva de que 'mejor que' es una relación asimétrica, transitiva, análoga a 'mayor que' - analogía que resulta importante en filosofía si comparamós la prueba de la existencia de Dios de San Anselmo, por ejemplo, con la de San Agustín. Esta segunda noción -dice Brumbaugh- sugiere de inmediato que la serie de números es un modelo formal adecuado para la comparación racional y que las alternativas son o tal cálculo o el rechazo de la pertinencia del pensamiento respecto a la acción. Si $A$ es mejor que $B$, queremos saber cuánto mejor, mejor para cuántas personas y mejor por cuánto tiempo. Y de modo intuitivo sabemos que estas preguntas tienen sentido". Pero estas dos nociones intuitivas: 1) que los números finitos no pueden aplicarse, por ejemplo, a la búsqueda socrática de la sabiduría o, lo que es lo mismo, a la propia hija a la que no se vendería a ningún precio, y 2) que los números finitos pueden aplicarse, por ejemplo, en la comparación, constituye una antinomia que parece irreconciliable. Encuentra su solución al expander la noción de números cardinales para que incluya los números transfinitos. Entonces las dos nociones - que los valores pueden ordenarse y compararse por medio de un modelo aritmético aditivo y que algunos valores no se relacionan de modo aditivo- resultan comparables. Brumbaugh dice que hay órdenes de valores, que están en la relación "mayor que" y que pueden representarse como teniendo los números cardinales $n$, $\chi_{0}$ y $\chi_{1}$, donde la relación entre $\chi_{0}$ y $\chi_{1}$ es que $\chi_{1}$ es $2^{0}$, es decir, la totalidad de valores de una cosa con $\chi_{0}$ propiedades. Según Brumbaugh, esto soluciona las cosas hasta el punto a que llegan las dos nociones citadas: mantenemos la comparabilidad, pero sin reducibilidad, entre los órdenes. En otras palabras, mantenemos valores finitos e infinitos y sin embargo podemos comparar unos con otros ya que la infinitud $\chi_{1}$ es mayor que la infinitud $\chi_{0}$ y esta última es mayor que el número finito $n$.

Introduzcamos ahora estas tres clases de cardinalidades a la axiología. Primero debemos entender la diferencia entre un número y su aplicación, por ejemplo, la diferencia entre el número 2 y dos manzanas. Dos manzanas aplica el número 2 a dos cosas que son manzanas. En general, puede haber

18 "Changes of Value Order and Choices in Time", en Value and Valuation: Studies in Honor of Robert S. Hartman, The University of Tennessee Press, Knoxville, Tenn., 1972, pp. 49-61. 
una infinidad de parejas de cosas, pero sólo hay un número 2. Por esta razón, Russell define el número 2 como la clase de todas las parejas. Define el número extensionalmente: cualquier par es un elemento de la extensión del número 2, y este último es un concepto. También en axiología debemos hacer la distinción entre el número como concepto y los números en aplicación. Así, cuando decimos que una cosa tiene dos propiedades, aplicamos el número 2 a las propiedades de la cosa, pero el número axiológico 2 se refiere a los conceptos de estas propiedades, a dos predicados de una intensión, es decir, del contenido de un concepto. Así, de acuerdo con Russell, un número es la clase (o extensión) de clases (o extensiones) similares a una clase dada (o extensión), es decir, en el caso de pares, una correspondencia de uno-a-uno con cualquier par. También en la axiología formal tenemos una correspondencia de uno a uno, por ejemplo, entre cualesquiera cosas buenas. Tienen en común que posen "todas" sus propiedades, digamos $p$. Todo lo que es bueno tiene el número $p$ de propiedades, que resulta ser $\chi_{0}$ aplicado a ella. Este número mismo es el valor "bueno". Se refiere a la totalidad de intensiones similares a una intensión dada $-\mathrm{y}$ esta totalidad es precisamente el valor "bueno". Los valores "menos que buenos" son las totalidades de intensiones similares a intensiones menos que $p$. Si se da una intensión de $p$ predicados (un conjunto de predicados), que determina cierta extensión (clase), entonces las cosas que pertenecen a la extensión (los miembros de la clase) que tienen $p$ propiedades son tales cosas buenas (buenos miembros de la clase), y todas las que tienen menos que $p$ propiedades son menos que buenas (medianas, malas, etc.) Así como un número como 2 gobierna un conjunto de extensiones, es decir, un conjunto de conjuntos de cosas, por ejemplo, pares, asi un valor como bueno cubre un conjunto de intensiones, es decir, un conjunto de conjuntos de predicados.

Al hablar de predicados más que de propiedades, elevamos el valor de una cosa hasta el valor formal de un concepto, lo mismo que Russell, por su definición, elevó el número 2 como concepto por encima de todos sus usos cuando se aplica a pares. Así, pues, justo como un número es la clase de las clases similares a una clase dada, así un valor es la clase de intensiones similares a una intensión dada; y este valor no es ya del tipo de valor que experimentamos, es el puro concepto formal "valor" que corresponde al puro concepto formal "número". Este último es un conjunto de extensiones, el primero un conjunto de intensiones.

Una intensión es un conjunto de predicados, a saber, de las palabras que aparecen en la definición o exposición de un concepto. Por ejemplo, en el concepto "silla", las palabras "estructura cuya altura alcanza mi rodilla, con un asiento y un respaldo", constituyen la intensión que, de este modo, consiste de las palabras "altura que alcanza la rodilla", "estructura", "asiento" 
y "respaldo". Si bien es difícil distinguir los conjuntos de propiedades en las cosas, los conjuntos de predicados en las definiciones y exposiciones son fácilmente distinguibles. Lo único que necesitamos hacer es abrir un diccionario y buscar la definición de cualquier palabra que allí se dé. Esta definición lexicológica consiste de un número de palabras, y estas palabras pueden distinguirse y enumerarse unas tras otras.

Ahora estamos listos para aplicar los números $n, \chi_{0}$ y $\chi_{1}$, que significan conjuntos de intensiones, a la teoría axiológica. Las intensiones de un número finito de predicados se llaman definiciones, las intensiones de un número $\chi_{0}$ de predicados se llaman exposiciones y las intensiones de $\chi_{1}$ de predicados se llaman descripciones. Cada uno de estos tres tipos de conjuntos de predicados define un tipo especifico de conceptos; y el cumplimiento de cada uno de tales conceptos define un tipo específico de valor. Estos tipos de valor, llamados sistemáticos, extrínsecos e intrínsecos, respectivamente, corresponden a los tres tipos posibles de conjuntos de predicados, llamados finitos $(n)$, enumerables infinitos $\left(\chi_{0}\right)$ y no enumerables infinitos $\left(\chi_{1}\right)$.

Los conjuntos finitos intensionales (definiciones) definen conceptos formales (conceptos sintéticos en el sentido de Leibniz y Newton y de la Lógica de Kant). Las cosas que les corresponden son construcciones de la mente humana, como los círculos geométricos. Tales cosas o bien llenan su concepto o no son tales cosas; o son o no son lo que se dice que son. No hay círculos geométricos buenos o malos. Un círculo que carezca de una sola de las propiedades del concepto "círculo" no es un círculo. Así, pues, las construcciones de la mente humana sólo tienen dos valores, que llamaremos valores sistémicos: perfección o no valor. Este es el modelo de la valoración blanco y negro de las cosas, el tipo de valoración más simple que hay. Ya que pertenece a construcciones de la mente, es obvio que al aplicarse a las cosas reales las "prejuzga" -es el modelo del prejuicio. Este tipo de pensamiento se basa en la categoría lógica de limitación; la variedad del mundo se limita a sólo dos distinciones: A y no-A (es decir, blanco y no blanco, comunista y no comunista). Personas de este tipo están limitadas, bornées, ciegas a los valores, tal como es amusical una persona que sólo conoce dos tonadas, una es el himno nacional y la otra no. La valoración sistémica es el modelo del pensamiento esquemático y dogmático.

Los conjuntos enumerablemente infinitos de predicados intensionales (exposiciones) definen conceptos abstractos y categorias (conceptos analiticos en el sentido de Leibniz y Newton y de la Lógica de Kant). La abstracción "extrae" propiedades que son comunes cuando menos a dos cosas. Estas propiedades son denumerables, ya que deben abstraerse una por una (en el proceso de aprender a hablar; encontramos un ejemplo notable en la autobiografía de Helen Keller), pero hay una infinidad de tales propiedades po- 
sibles. Los referentes de tales conceptos son las cosas del mundo cotidiano. Cada una de estas cosas tiene en potencia un número infinito de propiedades en común con otras cosas tales — según la extensión de la clase--, pero en la práctica la valoración entregará sólo unas cuantas de estas propiedades; como hemos visto, una cosa con diez propiedades consideradas para la valoración tiene $2^{10}-1=1023$ valores posibles, $\mathrm{y}$ un vaso de vino de Borgoña que, de acuerdo con un experto tiene $15^{8}$ propiedades, tiene $3.6 \times$ $10^{46}$ valores posibles. Lo que se valora no es la cosa en sí misma, sino su posesión de los predicados de clase. El que una cosa llene un concepto abstracto constituye un valor extrinseco. La valoración extrínseca es el modelo del pensamiento pragmático cotidiano.

Los conjuntos no enumerables de predicados intensionales (descripciones) definen conceptos singulares (también axiomas). Las cosas que corresponden a tales conceptos son únicas. La unicidad es la contrapartida intensional de la singularidad extensional. Los predicados de tales intensiones pueden ser palabras de significado infinito, es decir, palabras que pueden significar cualquier otra palabra del lenguaje. Tales palabras son metáforas. Una metáfora es un conjunto de predicados usado como una variable. Por ello puede sustituir, en principio, a cualquier otra palabra del lenguaje -y aun a sí misma como palabra común más que como metáfora, como en "la crema de la crema". Dado que la totalidad de todos los lenguajes comunes posibles tiene $\chi_{0}$ palabras, cada una de las cuales puede significar como metáfora $\chi_{0}$ sentidos o significados, el significado total del lenguaje metafórico es de ${ }_{2} \chi_{0}=\chi_{1}$, significados. Por lo tanto, el lenguaje metafórico es una infinitud no enumerable, un continuo. Dado que un elemento de un continuo puede ser él mismo un continuo, una metáfora puede ser ella misma un continuo. Una conjunción de un número finito de metáforas es un poema o una novela poética. El que una cosa llene un concepto singular, entendido en este sentido, constituye un valor intrinseco. El valor intrínseco es la valoración de los poetas y artistas, de los amantes y los místicos, de los magos y publicistas, de los grandes cocineros y los políticos, de los teólogos y los científicos creadores. Es una valoración enfática -y empática.

El valor sistémico, el valor extrínseco y el valor intrínseco, son las tres dimensiones axiológicas. Forman, como explica el profesor Brumbaugh, una jerarquía de riqueza, pues el valor intrínseco es más rico en cualidades o mejor que el valor extrínseco, el valor extrínseco más rico en cualidades o mejor que el valor sistémico y, a la inversa, el valor sistémico más pobre en cualidades o peor que el valor intrínseco. Cuando estos conceptos axiológicos se aplican a una cosa real, miden el valor de la cosa — justo como el número 2 aplicado a un par toma la medida de este par en cuanto tal. Así como el número 2 es menor que el número 3 y el 3 mayor que el 2, así las aplica- 
ciones de estos números a pares y tríos respectivamente miden a tales pares y tríos: un trio es mayor que un par y un par es menor que un trío. Dado que los conceptos axiológicos, más que los valores de las cosas, son conjuntos de predicados o palabras, más que conjuntos de propiedades, decimos que los conjuntos de predicados o palabras miden los conjuntos de propiedades o cosas. Llamamos a esto, en la axiología formal, la estructura axiométrica de la intensión. En otras palabras, la definición o significado de algo mide el valor de la cosa en cuestión. El significado de "silla", es decir, los cuatro predicados en el concepto de silla, mide el valor de las sillas reales, que tienen o no las propiedades correspondientes.

\section{La estructura axiológica de la personalidad}

Ahora estamos listos para aplicar la axiología formal a la psicología, en particular a la personalidad humana, aplicando las tres dimensiones axiológicas, sistémica, extrínseca e intrínseca al ser humano. La definición axiológica de un ser humano es: un ser humano es ese ser que tiene su propia definición de si mismo en si mismo. Una silla no sabe que es una silla, pero yo sé que soy yo. No importa cuál sea él aspecto de la gente de Marte - por lo que sabemos, podrian parecer sillas - si pueden decir "yo" y reflexionar sobre ello, son seres humanos.

De esta definición de un ser humano se siguen consecuencias de largo alcance. Cuando pienso en mí, soy tanto sujeto como objeto. El sujeto, yo, piensa el objeto Yo mismo o Mí mismo. En "pienso en mí mismo" o "pienso en mí", en lo que se piensa es en mí mismo. Esto significa que el sujeto, yo, no es pensado sino que piensa. Podemos pensar en el $Y o$ que piensa en $M i$, sólo convirtiéndolo en un $M i$. Tenemos entonces " $Y o$ pienso en $M i$, pensando en $M i$ "'. Aparece ahora otro Yo. Al tratar de pensarlo, obtenemos: " $Y o$ pienso en $M i$, pensando en $M i$, pensando en $M i$ "', y aparece entonces un tercer Yo que no puede ser pensado -y asi ad infinitum. Por la mera definición de un ser humano como autorreflexionante, aparece como una infinitud. Nunca puedo apresarme a mí mismo como pensante. Lo peculiar es que uno de los primeros tratados matemáticos sobre la infinitud, el de Dedekind (1887), usó este ejemplo para demostrar la existencia de sistemas infinitos. Royce, doce años después, volteó la prueba de Dedekind a fin de demostrar la infinitud del ser humano. Axiológicamente, el ser humano es una infinitud real. Es más, es una infinitud no enumerable: ya que lo que es cierto acerca de $Y o$ y de $M i$ es cierto acerca de cualquier pensamiento que pueda tener. Si pienso en esta silla, puedo pensar en mi pensando esta silla, y en mi pensar pensando esta silla, y así ad infinitum. Así, cada uno de mis pensameintos puede constituir una infinitud. Si puedo tener una infinitud enumerable de pensamientos -como puedo en potencia-, enton. 
ces la totalidad de esta infinitud de pensamientos es no enumerable, pues ${ }_{2} \chi^{0}=\chi_{1}$.

Ya que, de acuerdo con nuestra definición, una infinitud no enumerable es un valor intrínseco, el ser humano es un valor intrínseco. Es ésta una definición objetiva del valor de un ser humano. Si ahora definimos. lo bueno moral como la aplicación del valor intrinseco a los humanos, entonces la bondad en cuestión es la bondad moral.19

Veamos qué significa esto. Hemos dicho que una cosa es buena si tiene todas las propiedades que corresponden a la intensión de su concepto o, en breve, si cumple su definición. La definición del ser humano está en él mismo. En consecuencia, un ser humano es bueno cuando cumple con su propia definición de sí mismo. ¿Qué significa esto? Significa que es moralmente bueno si es como es. Todas las palabras de la ética significan esto mismo, esta identificación de mí mismo conmigo mismo: ser sincero, ser honrado, ser genuino, ser auténtico, ser fiel a mí mismo, tener integridad, tener respeto de mí mismo - todas estas palabras significan que yo soy como soy, que soy yo mismo. Esto parece algo muy sencillo y, sin embargo, es dificilísimo de alcanzar. Ya que puedo definirme a mí mismo de las tres maneras, sistémica, extrínseca e intrínsecamente.

Cuando me defino sistémicamente, me propongo como un sistema, construyo algo como yo mismo que no soy yo. Vivo como algo construido. Karen Horney llama a esto el "sistema del yo". Conduce, a colapsos neuróticos y a enfermedades del yo; pues a la larga no podemos "vivir" como una construcción imaginaria.

Pero una persona puede definirse también extrinsecamente, como un miembro de alguna clase. En nuestras vidas estamos de continuo en situaciones externas, desempeñando toda clase de papeles. Soy un padre, un empleado, un rotario, un comelón, un orador, un dormilón, un conductor, un profesor, etc. Durante mi vida estoy en millones de situaciones. Pero la suma de estas situaciones no soy yo mismo. No tocan el núcleo de mí mismo que es mi definición intrínseca de mí mismo; no como esto o aquello, haciendo esto a aquello; sino como la Gestalt de mi ser esencial, simplemente como soy. ¿Quién soy? Yo soy yo. Esto, y ninguna otra cosa, es mi autodefinición. Por ello, a fin de cumplir con esta definición, tengo que ser yo mismo, es decir, ser fiel a mí mismo, sincero, honrado y tener todos los demás atributos morales mencionados. Soy, intrínsecamente, pura autorreflexión. Desde luego, ésta está revestida con un cuerpo humano. El cuerpo es, por así decirlo, el vestido terreno del Yo. Soy este ser humano en este planeta Tierra. Nací

19 Para mayores detalles, véase Robert S. Hartman, "Four Axiological Proofs of the Infinite Value of Man", Kant-Studien, LV, 1964, pp. 428-38. Reimpreso en Marvin C. Katz, Sciences of Man and Social Ethics, Boston, Branden Press, 1969, pp. 29-45. También, La estructura del valor, p. 300 n., y The Structure of Value, Carbondale, Ill., 1969, p. 116. 
como un infante desnudo y tengo que morir. En éste el punto capital de ser yo mismo en esta Tierra; y el ser profesor o cualquier otra cosa extrínseca es algo diferente a ser este humano, nacido en este planeta Tierra y que tiene que morir. En realidad, una definición extrínseca de mí mismo no es la definición de mí mismo.

A fin de cumplir intrínsecamente con la definición de mí mismo no debo ni construirme a mí mismo ni aun abstraer de mí mismo, sino simplemente SER, a saber, identificarme a mí mismo conmigo mismo. Esta es la tarea más difícil e importante de mi vida moral. Es difícil ser simplemente, ser natural y sin pretensiones, sin enorgullecerse ni avergonzarse de nada. Algunas veces lo logramos, "dejándolo todo", y saliendo de vacaciones, estando a solas con nosotros mismos y volviendo a conocernos, "poniendo de nuevo nuestras almas en nuestro cuerpo" (Paul Weiss). Por así decirlo, ser moral es poner el espíritu de las vacaciones en nuestra vida cotidiana. En este sentido, la moral se manifiesta a sí misma cuando no tratamos de impresionar a nadie ni positivamente con nuestros logros, ni negativamente con nuestros fracasos. Es lo que hace que los niños y los perros nos amen y que nuestra mujer nos contemple mientras dormimos. Solamente ser, en la vida cotidiana, es la mayor madurez. Es también algo muy poderoso, ya que pone en juego la infinitud de nuestro ser intrinseco.

Así, pues, la auto identificación es justo ser uno mismo. Axiológicamente, significa la auto definición intrinseca.

Así, la estructura axiológica de una personalidad es la aplicación de las tres dimensiones axiológicas - -sistémica, extrínseca e intrínseca - a la auto definición de un ser humano. Significa que yo puedo verme a mí mismo tanto sistemática o extrínsecamente cuanto intrínsecamente o, en otras palabras, que yo puedo concebir o sentir a mi yo en cualquiera o en las tres dimensiones axiológicas. De modo correspondiente, hay tres aspectos del yo, en realidad tres yo: el yo mental o de la mente, el yo social o la función en sociedad y el verdadero Yo o mi identificación conmigo mismo (más que con mi función en la sociedad o con mi inteligencia). Las tres dimensiones axiológicas del hombre son pues Pensamiento, Sociedad y Yo. Su interjuego es el patrón dinámico axiológico de la personalidad.

Podemos simbolizar este patrón total como un cono invertido con una pequeña bandera en la parte superior (véase p. siguiente) .

El Yo es la dimensión vertical dentro de nosotros. Por ello hablamos de psicología "profunda". Tenemos una profundidad infinita porque podemos aclarar nuestra autoconciencia con claridad infinita - con completa transparencia. Mientras más lo hacemos, más nos convertimos en nosotros mismos y de más poder dispondremos en el mundo. E1 Yo es nuestra reserva de poder. Tenemos un poder infinito a una infinita profundidad dentro de nosotros. No es fácil hacer fluir este poder. Sólo podremos hacerlo si nos li- 


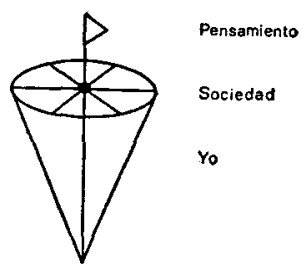

bramos, por así decirlo, de la dimensión horizontal, la de la sociedad, y hasta de la pequeña bandera de la parte superior, el pensamiento. Somos como icebergs. La mayor parte de nosotros está sumergida; y la parte horizontal está en la superficie, nada en el agua como la punta de un iceberg o, lo que es mejor, como un lirio acuático, muy frágil. Esta parte horizontal es nuestro yo social, el Mí mismo más que el Yo. Se conecta socialmente, con otros mí mismos iguales - y la red de conexiones se llama Sociedad. Así, tenemos la dimensión horizontal de la sociedad cuando tenemos trato social con la gente, y tenemos la dimensión vertical cuando podemos decir que tenemos trato con nuestro propio Yo. Son éstas las dos dimensiones de nuestra personalidad, la social y la existencial; a esta última la llamamos la dimensión humana o espiritual del Yo interior.

Existe aún una tercera dimensión -la intelectual-, que construye sistemas y los impone a la sociedad y con frecuencia al Yo. Es nuestra dimensión sistémica. Los alemanes son particularmente aptos en ella. Llevan lo sistémico a sus últimas consecuencias - la disciplina es su valor más alto- o lo fue, aunque significara el fin del mundo. Leemos acerca de Eichmann y la "banalidad del mal". ¿Fue Eichmann un malvado? No, en su opinión. Sólo cumplió con su deber. Afirmó: "Nunca maté a nadie. Soy un experto en transportes. Mi trabajo consistía en preparar horarios - ho rarios de trenes. Eso fue lo único que hice. Cumplí las órdenes que se me dieron. No me importaba a dónde iban. Mis órdenes eran preparar horarios de trenes y eso fue ló que hice. Me ocupé de transportar gente." Bien, las transportó a los hornos, pero eso era incidental. Lo hizo muy concienzudamente y se sentía orgulloso de ello. Era un hombre sistemático y el sistema era su vida. En su celda de Jerusalén daba todas las mañanas, a la misma hora, tantos pasos en una dirección y tantos en otra. Fumaba exactamente cuatro cigarrillos al día. Limpiaba su piso dando exactamente el mismo número de trapazos cada día. Una vez que se le interrumpió y perdió la cuenta, estuvo alterado todo el día. El orden era su valor, en ambos sentidos de la palabra. Sus trenes cumplían con el horario y eso era lo único que le importaba. Podemos decir que esto es alemán. Pero, ¿qué decir acerca 
de esto? "Soy el capitán de un submarino que tiene 16 bombas nucleares. Cada una de ellas tiene más poder ígneo que todas las bombas que se hayan tirado en todas las guerras juntas - cada una de ellas. $\mathrm{Y}$ al recibir mis órdenes, apretaré el botón y dispararé mis bombas. Ese es mi deber. Esa es la razón de mi vida. Cumplo órdenes. Soy un capitán de un submarino Polaris. Transporto fuego hacia la gente. Soy miembro de un sistema y hago lo que el sistema me dice que haga." ¿Qué diferencia tiene con Eichmann? Ninguna. Mientras seamos miembros de un sistema haremos lo que el sistema nos diga.

Este es el valor sistémico. Es amoral - como lo son la ley y la ciencia. Ambos son sistemas. Esta amoralidad puede ser inmoral, pero también puede ser moral. Todo depende de cómo se use el sistema. No podemos vivir sin valores sistémicos - pero no debemos exagerarlos. Todos los otros valores son más importantes que los valores sistémicos, por importantes que éstos sean. La vida está llena de valores sistémicos. Pero a veces, los valores sistémicos entran en colisión con nuestra propia individualidad. La gente tiene buen sentido de ello, pero no lo bastante bueno. Se rebela contra los nuevos números telefónicos, pero no contra las bombas nucleares. $\mathrm{Al}$ parecer, está empezando a rebelarse contra los jets, dado que las compañias aéreas pierden dinero a causa del poco tráfico. Un avión es una construcción de metal -está allá arriba por las puras leyes físicas. Tiene un millón de partes ensambladas por el hombre, y el hombre simple y sencillamente no está a la altura de la perfección de los sistemas. De tiempo en tiempo, algo sale mal. De eso viven las compañías de seguros. Viven del hecho de que el hombre es muy bueno sistémicamente, pero no lo bastante bueno. La mayoría de los aviones se mantiene en el aire, pero caen los suficientes para que las aseguradoras hagan negocio. Las compañías de seguros quebrarian, a) si ningún avión se cayera nunca y $b$ ) si todos se cayeran. Hay un balance muy sutil en el universo entre lo perfecto y lo imperfecto, que puede ser calculado por medio de las leyes estadísticas. La mayoría de la gente está viva, pero todos tenemos que morir algún día. La mayoría de nosotros está sana, pero $1 / 64$ por ciento estará enfermo mañana. Las catástrofes no forman parte de nuestro contrato de seguro - siempre aparece en tipo menor la cláusula sobre los desastres de force majeur, los llamados "actos divinos" que más parecen obra del demonio. Podemos saber con exactitud no a quién va a sucederle algo, pero sí que algo va a suceder. Todos los automóviles marchan bien por la carretera, pero sabemos que cada fin de semana mueren $5^{8} 5$ conductores. Después aparece la estadística real y resultan 586 . Nadie quiso que fuera así. El mundo es perfecto, pero tiene una pequeña falla de contingencia, y no es tan perfecto. Simplemente, la inteligencia humana no está a la altura de la perfección completa. 
Esta es la dimensión estadística y sistémica del valor. Es muy necesaria. Cuando nuestra esposa va al sanatorio a tener un niño, se le da un número a ella y al niño también, y uno espera que ambos tengan el mismo número. Lo sistémico es muy importante para cosas muy importantes de nuestra vida, pero no es lo más importante.

Pasemos ahora a la dimensión social. Aquí nos reunimos con nuestros llamados prójimos en la llamada sociedad para una llamada participación. Quienes trabajan en compañías de seguros se reúnen - pero sus esposas raras veces los acompañan, ya que no entenderian de qué se trata. Y si nuestro agente de seguros entrara en una sociedad filosófica, no entenderia mucho (y viceversa). Así, el mundo está dividido socialmente en clases; y cuando Karl Marx habló de clases se refirió a clases sociales, pero son también clases lógicas. Las personas son clasificadas, cada una en su propia sección, y valorada como esto o aquello. Damos a esto el nombre de valor social o extrínseco. Se nos valora extrínsecamente. Se nos valora como buenos agentes de seguros, buenos maestros, buenos empleados. Por lo común, los valores monetarios son valores extrínsecos. Son valores limitados - nadie vendería a su hijo, ni compraría una mujer. El nivel del valor intrínseco que está en mi propia profundidad, es infinitamente más importante que todos los sistemas y todo el dinero del mundo.

Demos ahora un ejemplo de cómo los tres niveles de valores van juntos. Digamos que un joven entra en una tienda a comprar un paquete de cigarrillos. Hay una vendedora joven a la que dice, "Deme unos Marloboros". Ella le responde, "Aquí los tiene”. "¿Cuánto?”, pregunta él. “5 pesos”, responde ella. La joven entrega el paquete, él le da los 5 pesos, le dice adiós, ella responde adiós y gracias. ¿Qué clase de relación es ésa? Una relación puramente sistémica, un contrato legal de venta. Él entregó 5 pesos y ella el dio un paquete de cigarrillos. Podía haberse tratado de una máquina expendedora, no se necesita una muchacha para eso. Ya hay máquinas que hasta dicen "gracias".

No es mucho lo que entra en una relación sistémica. Al día siguiente el joven entra en la misma tienda y encuentra a la misma vendedora y le dice, "Unos Marloboros". Ella responde, "Oh, sí, usted quiere Marloboros -5 pesos". Y él dice, "Sí, 5 pesos", y la mira. "Por Dios -piensa—, si es una muchacha". Y la vuelve a mirar y piensa que es bonita. Le dice entonces, "¿Cuánto tiempo hace que está aquí?" Ella contesta, "Tres meses", y él le dice, "¿De dónde es usted?" "De Guadalajara". Antes de que pase mucho tiempo, han hablado del tiempo, desde luego, y después de los respectivos padres, etc. Entran en conversación. También es una relación, un dar y tomar, pero es una relación social, no legal. Han pasado de la relación sistémica, que sólo tiene una propiedad, a la social que puede tener una infinidad de propiedades. Pues pueden hablar todo un día y muchos más. 
En la tienda hay un asiento, y el joven se sienta a verla trabajar y, cuando no hay clientes, habla con ella. Un día le dice, "Vamos a cenar juntos", lo hacen y lo pasan muy bien. Han añadido algo a su relación, ésta se ha enriquecido. También podemos definir el valor como un enriquecimiento de propiedades. Mientras más rico en propiedades sea algo, más valor tendrá. Mientras más pobre en propiedades sea algo, menos valor tendrá. Ellos han enriquecido sus propiedades. Enriquecimiento de propiedades -es lo que obtienen esa noche y otras iguales; están enriqueciendo sus relaciones de continuo - hasta que abarca todo su ser. Y un día, al encontrarse, él le pregunta —o ella lo hace_, "¿Cuándo nos casamos?”, y de pronto están frente a un sacerdote y se prometen fidelidad mutua por toda la vida.

Han caminado un largo camino desde el contrato de venta. Ahora son marido y mujer, y un matrimonio verdadero, el amor, nada tiene que ver con una relación social. Es una relación de núcleo interno a núcleo interno. El amor es esta relación.

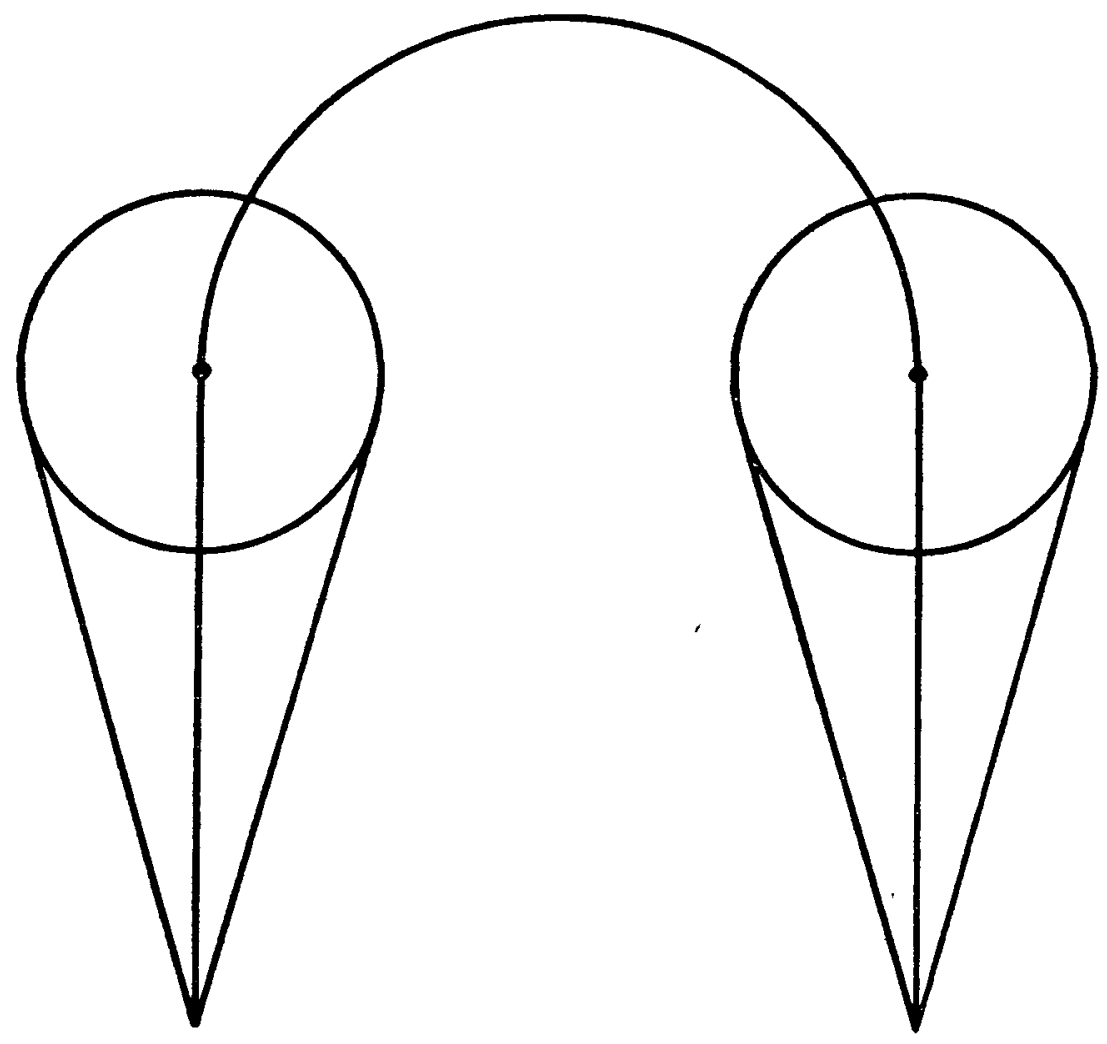


¿Cómo saben que se aman uno a otro? Porque han entrado en sí mismos, han indagado allí dentro y se han preguntado a sí mismos, ¿Quién es esta persona? - no ¿Qué es esta persona? Él ve a esa muchacha de dieciocho años como abuela de sus propios nietos - cuando sus hijos no han sido concebidos aún. El Yo interior salta por encima del espacio y del tiempo, ve toda la vida con una sola mirada. La característica principal del Yo interior es la intuición inmediata. Nace de la transparente claridad de uno mismo.

\section{La infinitud del Yo}

Recordemos nuestra definición original del Yo. Yo tengo un valor moral en la medida en que cumplo mi propia definición de mí mismo. Esta definición es: "Yo soy yo". En la medida en que yo soy yo, soy una persona moralmente buena. La bondad moral es la profundidad del propio ser del hombre. Es la mayor bondad del mundo, la buena voluntad de Kant. Y si cada uno de nosotros y cada una de las personas del mundo fuera ella misma y siguiera su propio yo interior o, como decimos, la voz de su conciencia, todo se arreglaría y todos los problemas se resolverían. No escucharíamos a los falsos profetas, a los políticos, a quienes quieren usarnos para su propia ambición. Sólo seríamos, y seríamos nosotros mismos. Conoceríamos los verdaderos valores. Como dice Larissa —en el $D r$. Zhivago, de Pasternak - a su amigo Zhivago, ya muerto, al final del libro: "El enigma de la vida, el enigma de la muerte, el encanto del genio, el encanto de la belleza sin adornos - todo esto fue nuestro. Pero las pequeñas preocupaciones de la vida práctica - cosa como la remodelación del planeta -, gracias, eso no es para nosotros". ¿Cosas pequeñas como la remodelación del planeta? Todo el mundo es nada en comparación con el Yo infinito, el enigma de su vida y de su muerte, su conciencia de la belleza, su genio.

Esto no quiere decir que no debamos ser sociales $n i$ inteligentes. Por el contrario, mientras más seamos nosotros mismos, más poder tanto social como intelectual tendremos. Una persona verdaderamente inteligente es la que puede movilizar sus recursos internos, el infinito poder que hay dentro de ella, para manejar su propia vida. Un intelectual sólo es una persona que sabe cómo pensar.

El primero que adivinó el valor infinito del hombre fue el salmista que dijo que el hombre fue hecho sólo un poco menor que los ángeles. El primero en articular este valor infinito de la persona humana fue Jesús. Vio y demostró el valor infinito de lo individual. Muchos individuos han sido capaces de reunir los recursos infinitos de su Yo interior en épocas de crisis y desastres. Hemos dicho que "Yo soy yo" no es una identidad, sino una predicación. De esto se sigue una segunda prueba lógica de la infinitud del Yo, además del regreso al infinito del Yo que éncontramos en Royce 
y otros. Podemos dar a esta nueva prueba el nombre de prueba a partir de la identidad. Si el segundo "yo" en "Yo soy yo" es el predicado del primero, entonces es el concepto cuyo sujeto es el primero. Así, pues, el primero es cualquier momento de mi carrera en el espacio y el tiempo. El segundo es lo que hace una persona a partir de las fracciones infinitas de mi vida en el espacio y el tiempo. Todos estos momentos tienen que reunirse para que yo sea la misma persona que era cuando niño, cuando adolescente, ahora cuando hombre y pronto cuando viejo. Todos estos momentos deben pertenecerme, a la misma persona. Debemos poder decir: yo nací hace $4^{0}$ años en tal o cual lugar. Y soy yo quien está aquí ahora. Así, el segundo yo reúne nuestros momentos en el espacio y el tiempo. Es el concepto que hace de mis momentos espacio temporales un todo.

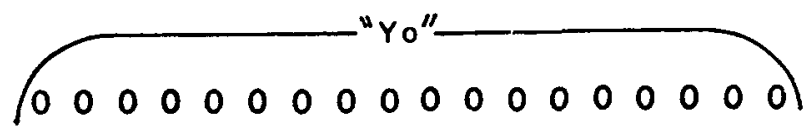

Yo al nacer

Yo ahora

Yo al morir

Existen tribus primitivas que son como infantes que no saben decir "yo". Cuando Molubabeba habla a Malinowski de su infancia no le habla de sí mismo ni de "yo", sino de "Molubabeba niño". otro ser. Se necesita el pensamiento abstracto para apresar juntos nuestros momentos en el tiempo. Para él el sol de hoy es un sol distinto al de ayer. "Yo" es mi concepto de identidad en el tiempo y el espacio. La infinitud de los momentos de nuestra vida no empieza sólo con nuestro nacimiento. Empieza con nuestra concepción. Pero una vez que hemos ido tan lejos no necesitamos detenernos allí. En realidad empezamos con nuestro padre y nuestra madre. Pero también ellos tuvieron que ser concebidos, así que no empezamos tampoco alli, sino con nuestros abuelos y así retrospectivamente; en realidad empezamos con el inicio de la creación. De ello habla Walt Whitman en "The Song of Myself"

Para mí se hicieron preparativos inmensos,

llenos de fe y amistosos los brazos que me han ayudado.

Los ciclos guiaron mi cuna, boga que te boga como remeros alegres,

y las estrellas me dieron alojamiento en sus anillos

y mandaron influencias que cuidaran lo que habia de sostenerme.

Generaciones me guiaron antes de nacer del vientre de mi madre, mi embrión nunca fue torpe, nada pudo ahogarlo

Por él las nebulosas se unieron a un orbe, largos y lentos estratos se apilaron para que descansara, 
vegetales inmensos le dieron sustento,

saurios monstruosos lo transportaron en sus fauces y lo depositaron con [cuidado.

Todas las fuerzas han sido utilizadas sostenidamente para formarme [y deleitarme.

y ahora estoy aquí de pie en este punto con mi alma robusta.

Soy un resultado de la evolución. Whitman no conoció esa palabra. Soy el resultado de la creación. Empecé en la infinitud - y ¿dónde acabaré? ¿ Acabaré con mi muerte? ¿No están ahí mi hijo, mis nietas? Soy un eslabón en la cadena de generaciones sobre la tierra. Es más, soy inmortal. Mi Yo, mi espíritu - dijimos - no está en el espacio y el tiempo. ¿Cómo puede morir en el espacio y el tiempo? No puede. El cuerpo y la mente pueden decaer, pero el espíritu, mi conciencia de mi propio Yo dentro del todo de la creación, debe continuar. Vengo de la infinitud, voy a la infinitud. Goethe, el poeta alemán, dijo: "Soy inmortal en la medida en que he vivido inmortalmente" - en que he vivido completamente consciente en la plenitud de mi propia conciencia. Soy un prodigio de la creación - Whitman tiene razón. $Y$ si vivo como un vegetal, defraudo a la creación.

Así, cuando digo "Yo", comprendo a toda la creación. Kierkegaard dice que yo estoy anclado con mi propio Yo en la fuente de la creación misma. No sólo expreso los muchos momentos infinitos de mi vida terrena, expreso todo el lapso de toda vida entre la infinitud que fue y la infinitud que será.

Sólo los santos pueden vivir plenamente este alcance infinito del Yo. Un santo es una persona que pone todo su poder, todos los recursos de sí mismo, en su propia bondad, un hombre que ha descubierto su unidad con toda la creación, todos los hombres, todos los animales, aun todas las cosas. Vive en la profundidad de todos y de todo. Es un hombre de infinita compasión. La bondad intrínseca más profunda es vivir tan profunda y transparentemente dentro de nosotros, que vivamos profunda y compasivamente dentro de todo ser humano, aun dentro de todo ser vivo - de todo ser. Como le dijo San Francisco al Hermano León cuando éste trató de apagar el fuego del sayal del santo: "Hermano León, ten cuidado con el hermano fuego". O como Albert Schweitzer, quien se duele de tener que matar las bacterias al hacer una operación. La compasión es una de las piedras de toque del valor moral.

Otra es la Conciencia. Como la compasión, es un síntoma de la infinitud del Yo. Hemos hecho algo malo, nadie nos vio, no había ni un alma por ahí. Pero al volver a donde hay gente, sentimos que todos saben lo que hemos hecho. Una mala conciencia significa que tememos que todos tengan con-ciencia con nosotros. La forma clásica de este sentimiento que todos conocemos es Crimen y castigo de Dostoievski. Raskolnikov, un estu- 
diante, asesina por dinero a una vieja perversa. E's el crimen perfecto $y$, sin embargo, siente que todos lo saben, que todas las cosas lo traicionan. Por último se entrega, incapaz de vivir con su culpa.

Ahora bien, ¿por qué tenemos el sentimiento de que todos, de que todas las cosas, lo saben? Porque, como la compasión, en la conciencia nuestro Yo vive en cada uno y en cada cosa. La conciencia nos exhorta negativamente a hacer lo que la compasión nos obliga a hacer en forma positiva. La conciencia es la otra cara de la moneda. Nos hace darnos cuenta, negativamente, de todos y de todo en la creación, como lo hace en forma positiva la compasión. El Yo interior, hemos dicho, no está en el espacio y el tiempo. ¿"Dónde" está entonces? En todas partes. En otras palabras, en el núcleo interno de nuestro Yo somos intrínsecamente uno con todo otro Yo. Los conos de nuestra Mismidad se encuentran todos en el vértice. Hay una comunidad, un núcleo, de toda la humanidad. Jesús llamó a este reino el Reino de Dios que está dentro de nosotros; Kant lo llamó el Reino de los Fines. En él, todos somos intrínsecamente uno; y cuanto hacemos algo malo todos lo han hecho con nosotros y a través de nosotros. En mi Yo intrínseco, todos han hecho la mala acción; y por ello temo que todos lo sepan. Todos lo han hecho, intrínseca, auque no extrínsecamente, conmigo; y todos lo saben, intrínseca, aunque no extrínsecamente. Ambas, la compasión y la conciencia, nos hacen darnos cuenta de nuestra unidad espiritual con toda persona y de nuestra responsabilidad hacia ella y de ella hacia nosotros.

En la compasión y la conciencia, el Yo intrínseco se llena con una infinitud de propiedades, enriqueciéndose a sí mismo infinitamente en profundidades cada vez mayores de ese darse cuenta. Lógicamente, esto significa que el concepto que es el segundo "Yo" en "Yo soy yo" se llena con un valor infinito y adquiere un significado infinito. Este valor infinito, este significado infinito del Yo es, finalmente, la esencia de la Personalidad. Es, como dijo el poeta alemán, "la mayor bendición del hombre en la tierra". En una palabra, la personalidad es el valor de una persona; es el significado del concepto "Yo". Vivir significa enriquecer este concepto concienzudamente con un significado cada vez más profundo, cada vez más amplio, hasta el fin de nuestros días. Y el carecer de este valor de los va. lores, de este significado infinito significa estar muerto espiritualmente aunque se esté físicamente vivo, la enfermedad hasta la muerte de Kierkegaard, el vacío existencial de Viktor Frankl y otros existencialistas psicológicos. Permitaseme terminar con estas palabras de Lawrence Durrel en Cuarteto de Alejandria: "Ni siquiera ahora me atrevo a decir que era estúpida o insensible; sólo que no era capaz de reconocer lo que ocurría dentro de sí misma ('la camara obscura del corazón'), no podía poner un marco preciso alrededor de la imagen aterradora de su propia insignificancia en el mundo de los hechos ordinarios. El abismo que parecía rodearla estaba 
compuesto por una cualidad - una falla de valor, una falla en la atribución de significado que mata la alegría -esto es en sí mismo sólo la moralidad interna de un alma que ha descubierto el camino real hacia la felicidad, cuya desnudez no se avergüenza de sí misma."

RoBert S. HaRTMAN

INSTTTUTO de INVESTIGACIONES Filosóficas

Universidad Nagional Autónoma de México 